\title{
DESIGN OF STEERABLE FILTERS FOR THE DETECTION OF MICRO-PARTICLES
}

\author{
Cédric Vonesch, Frédéric Stauber and Michael Unser \\ Ecole Polytechnique Fédérale de Lausanne, Switzerland
}

\begin{abstract}
This paper presents two contributions. We first introduce a continuous-domain version of Principal-Component Analysis (PCA) for designing steerable filters so that they best approximate a given set of image templates. We exploit the fact that steerability does not need to be enforced explicitly if one extends the set of templates by incorporating all their rotations. Our results extend previous work by Perona to multiple templates.

We then apply our framework to the automatic detection and classification of micro-particles that carry biochemical probes for molecular diagnostics. Our continuous-domain PCA formalism is particularly well adapted in this context because the geometry of the carriers is known analytically. In addition, the steerable structure of our filters allows for a fast FFT-based recognition of the type of probe.
\end{abstract}

Index Terms - Steerable filters, Principal-Component Analysis (PCA), micro-particle detection, molecular diagnostics.

\section{INTRODUCTION}

\subsection{Motivation}

The current evolution towards personalized medicine is creating a need for cost-effective, high-throughput yet patient-specific diagnostics solutions. In this context, the Swiss company Biocartis has developed a micro-fluidic system that can simultaneously test a given sample for the presence of a large number of different biological markers.

The system is built around circular micro-particles (see Fig. 1) that carry suitable biochemical probes in their central part. The type of probe is encoded at the periphery of each micro-carrier through a series of perforations. The readout of the assay is performed in time-lapse microscopy using two complementary optical modalities: the binding process between the probes and the markers is monitored using fluorescence imaging, while the particles are tracked using brightfield imaging.

Here we concentrate on the latter problem. Specifically, our goal is to detect each particle and to decode its perforations so as to determine the type of probe it carries. To this end, the orientation of each particle must be determined in an accurate and computationally efficient way. We have thus chosen to develop an algorithm based on steerable filters, because they can provide non-discretized directional information using only a finite number of correlation measurements.

Our approach can be divided into two parts, corresponding to Section 2 and Section 3 of the present paper. We first design a family

This work was funded in part by the Center for Biomedical Imaging and ERC Grant ERC-2010-AdG 267439-FUN-SP. of steerable filters that is tailored to the patterns of interest based on Principal-Component Analysis (PCA). Then we use the obtained filters within a fast convolution-based algorithm for determining the location, orientation and encoding of each particle.

\subsection{Existing work and contributions of this paper}

Steerable filters have been characterized from two different mathematical perspectives: functional analysis $[1,2,3]$ and the theory of Lie groups $[4,5]$. While the angular part of steerable filters is relatively constrained (it must be a finite Fourier series), its design for optimal orientation selectivity has been the subject of recent work in the context of wavelet design $[6,7]$. There is more freedom when designing the radial part of steerable filters, which we do in the present paper.

The idea of approximating a single template in a steerable basis has been studied independently by Perona $[1,2]$ and Hel-Or \& Teo [8], using continuous-domain formalisms; others have favored purely discrete formulations [3,9]. Note that [9] covers the case of multiple templates, but only in combination with a discrete set of rotations. Here we consider a completely isotropic formulation in the continuous domain.

The originality of our work stems from the following contributions.

1. We extend Perona's continuous-domain approach $[1,2]$ to multiple templates.

2. We establish the connection between the isotropic PCA problem that underlies [9] and our steerability-constrained PCA. Moreover our functional perspective puts the emphasis on the rotation invariance of the principal-component subspaces and on their application to the efficient directional analysis of images.

3. We apply our framework to the specific problem in molecular diagnostics described above, where the templates are known analytically. This constitutes a strong motivation for our continuous-domain formalism and allows for an analytic design.

\section{DESIGN OF THE STEERABLE FILTERS}

In this paper we use $(r, \theta)$ to denote polar coordinates in $\mathbb{R}^{2}$. We will also refer to spaces of finite-energy functions using notations of the form $L_{2}$ (domain, codomain). For scalar-valued functions (codomain $=\mathbb{C}$ ) the inner product is defined as $\langle f, g\rangle=$ $\int \overline{f(s)} g(s) \mathrm{d} s$, where the integral is taken over the domain and the bar denotes complex conjugation. 


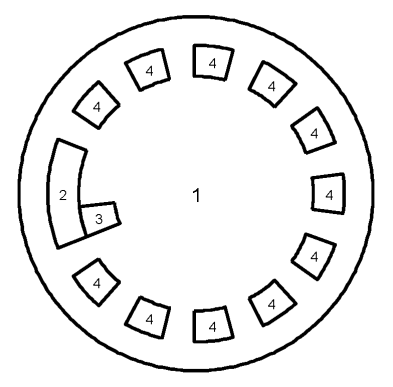

Fig. 1. Sketch of a particle (outer diameter: $40 \mu \mathrm{m}$ ). The numbers correspond to the indicator functions used in eq. (6).

\subsection{Characterization of steerable functions}

A function $\varphi$ is said to be steerable if its rotated versions can be expressed as a linear combination of a finite number of fixed basis functions, as in [10]

$$
R_{\theta}\{\varphi\}=\sum_{n=1}^{N} c_{n}(\theta) \varphi_{n},
$$

where $R_{\theta}\{\varphi\}\left(r, \theta^{\prime}\right)=\varphi\left(r, \theta^{\prime}-\theta\right)$. This is extremely useful for directional image analysis: one only needs to compute a finite number of convolutions (with the basis functions), from which convolutions under arbitrary orientations can then be interpolated using the expansion coefficients $c_{n}(\theta)$.

Equivalently, a steerable function can be characterized as a function that lives in a finite-dimensional subspace which is invariant to rotations [5]. We thus turn our attention to rotation-invariant subspaces.

Theorem 1. A finite-dimensional subspace of $L_{2}\left(\mathbb{R}^{2}, \mathbb{C}\right)$ is rotationinvariant if and only if it is spanned by functions of the form

$$
\varphi_{n}(r, \theta)=\phi_{n}(r) \mathrm{e}^{\mathrm{i} k_{n} \theta} \quad \text { where } \quad k_{n} \in \mathbb{Z} .
$$

A general proof of this result using the theory of Lie groups can be found in [5]. In the sequel we will focus on the choice of the integers $k_{n}$ and on the design of the radial components $\phi_{n}(r)$.

\subsection{From steerable PCA to isotropic PCA}

Classically, the principal components of a data set are defined in statistical terms: one constructs the direction that accounts for the largest variance, subtracts off this component from the data and reapplies the same scheme recursively.

A dual, approximation-theoretic formulation is to construct a sequence of subspaces by incrementally generating basis elements so as to optimally fit the data in a quadratic sense. The only practical difference with the first definition is that the mean of the data set is not necessarily subtracted before the analysis.

Here we will start from the latter interpretation. We will use the following notations: $\boldsymbol{f}=\left[\begin{array}{lll}f_{1} & \ldots & f_{M}\end{array}\right]^{T} \in L_{2}\left(\mathbb{R}^{2}, \mathbb{C}^{M}\right)$ is a vector-valued function representing a collection of desirable templates; $\varphi=\left[\begin{array}{lll}\varphi_{1} & \ldots & \varphi_{N}\end{array}\right]^{T} \in L_{2}\left(\mathbb{R}^{2}, \mathbb{C}^{N}\right)$ is a vector-valued function representing the principal components. Our goal is to minimize the following quantity, subject to the condition of Theorem 1.
Definition 1 (Steerable-PCA functional).

$$
J(\boldsymbol{\varphi})=\min _{\mathbf{C} \in \mathbb{C}^{M \times N}}\|\boldsymbol{f}-\mathbf{C} \boldsymbol{\varphi}\|^{2} \text { subject to }(1) .
$$

Note that in this paper inner products involving multi-valued functions should be interpreted according to the usual rules for the multiplication of scalars, vectors and matrices. For example, the norm of $\boldsymbol{f}$ is a scalar defined via $\|\boldsymbol{f}\|^{2}=\left\langle\boldsymbol{f}^{T}, \boldsymbol{f}\right\rangle$. In contrast, the notation $\left\langle R_{\theta}\{\boldsymbol{f}\}, u\right\rangle$ used below refers to a vector ${ }^{1}$.

Since the span of $\left(\varphi_{n}\right)_{n \in \llbracket 1, N \rrbracket}$ is constrained to be rotationinvariant we have $J(\varphi)=J\left(R_{\theta}\{\varphi\}\right)$, where $R_{\theta}$ is a rotation operator corresponding to an arbitrary angle $\theta$. If we average this relation over all angles and exploit the fact that $R_{\theta}$ is linear and unitary, we obtain the following equivalent expression.

Definition 2 (Isotropic PCA functional).

$$
J(\boldsymbol{\varphi})=\frac{1}{2 \pi} \int_{-\pi}^{\pi} \min _{\mathbf{C} \in \mathbb{C}^{M \times N}}\left\|R_{\theta}\{\boldsymbol{f}\}-\mathbf{C} \boldsymbol{\varphi}\right\|^{2} \mathrm{~d} \theta .
$$

\subsection{Eigen-analysis of the problem}

In this section we minimize the isotropic functional (2) without explicitly enforcing the steerability condition (1). Despite this seemingly more general setting, we shall see that our minimizer always generates a rotation-invariant subspace.

Definition 3 (Data operator).

$$
\begin{aligned}
\boldsymbol{T}: \quad L_{2}\left(\mathbb{R}^{2}, \mathbb{C}\right) & \rightarrow L_{2}\left([-\pi, \pi], \mathbb{C}^{M}\right), \\
u(\boldsymbol{x}) & \mapsto \boldsymbol{T}\{u\}(\theta)=\left\langle R_{\theta}\{\boldsymbol{f}\}, u\right\rangle .
\end{aligned}
$$

The operator $\boldsymbol{T}^{*} \boldsymbol{T}$ plays the same role as the correlation matrix that must be diagonalized in discrete PCA formulations. Perona's observations (Section III of [2]) are also valid in our case: $\boldsymbol{T}$ is compact and therefore $\boldsymbol{T}^{*} \boldsymbol{T}$ admits a discrete spectrum.

Definition 4 (Principal components). The (orthonormal) eigenfunctions of $\boldsymbol{T}^{*} \boldsymbol{T}$ are denoted by $\left(u_{n}\right)_{n \in \mathbb{N}}$, with the convention that the corresponding eigenvalues $\lambda_{n} \in \mathbb{R}^{+}$are ordered by decreasing magnitude: $\lambda_{0} \geq \lambda_{1} \geq \lambda_{2} \geq \ldots$

Property 1. If $\varphi_{n}=u_{n}$ for every $n \in \llbracket 1, N \rrbracket$ then $\varphi$ is a minimizer of (2).

Due to space constraints the proofs of the results given in this paper will be published elsewhere.

\subsection{Computation of the principal components}

The next result provides an algorithm for computing the basis functions $u_{n}$. Observe that they have the steerable form described in (1).

Property 2. The principal components can be obtained via the following quantities.

1. The multipole decomposition of the templates:

$$
\boldsymbol{f}_{k}(r)=\frac{1}{2 \pi} \int_{-\pi}^{\pi} \mathrm{e}^{-\mathrm{i} k \theta} \boldsymbol{f}(r, \theta) \mathrm{d} \theta
$$

\footnotetext{
${ }^{1}$ Note that complex conjugation is still applied to the left-hand argument.
} 


\section{The Hermitian-symmetric matrices}

$$
\mathbf{H}_{k}=4 \pi^{2} \int_{0}^{+\infty} \overline{\boldsymbol{f}_{k}(r)} \boldsymbol{f}_{k}^{T}(r) r \mathrm{~d} r .
$$

3. Their eigenvectors $\left(\mathbf{v}_{n}\right)_{n \in \mathbb{N}}$, ordered by decreasing eigenvalues $\lambda_{0} \geq \lambda_{1} \geq \lambda_{2} \geq \ldots$; we denote by $\left(k_{n}\right)_{n \in \mathbb{N}}$ the indices of the corresponding matrices $\left(\mathbf{H}_{k_{n}}\right)_{n \in \mathbb{N}}$.

4. Finally the eigenfunctions

$$
u_{n}(r, \theta)=\mathbf{v}_{n}^{T} \boldsymbol{f}_{k_{n}}(r) \mathrm{e}^{\mathrm{i} k_{n} \theta} / \sqrt{2 \pi \lambda_{n}} .
$$

\section{DETECTION OF THE MICRO-PARTICLES}

In the second part of this paper we discuss the application of our filter-design scheme to the pattern-recognition problem described in Section 1.1.

\subsection{Analytic construction of the steerable filters}

A typical set of micro-particles is depicted in Fig. 3. Up to rotation, each particle can be described by the function (see also Fig. 1)

$$
p=A f_{1}+B R_{\pi}\left\{f_{2}\right\}+B R_{\pi \pm \alpha}\left\{f_{3}\right\}+B \sum_{\ell=-5}^{5} c[\ell] R_{\frac{2 \pi \ell}{13}}\left\{f_{4}\right\},
$$

where $A$ and $B$ are brightness constants, $\alpha$ is a small fixed angle and the coefficients $c[k] \in\{0,1\}$ represent the code.

The symbols $f_{1}, \ldots, f_{4}$ represent indicator functions of the following geometric shapes, respectively: a disk; a long perforation which serves as an angular origin for reading the code; a short inner perforation which indicates in which direction to read the code; and small outer perforations corresponding to the code itself. Note that the small inner perforation is necessary because particles can flip upside down, which amounts to reflecting their code. These four indicator functions all have the general form given below.

Definition 5 (Templates).

$$
f_{m}(r, \theta)=\mathbb{1}_{\left[R_{m}, S_{m}\right]}(r) \times \sum_{k \in \mathbb{Z}} \mathbb{1}_{\left[-\frac{T_{m}}{2}, \frac{T_{m}}{2}\right]}(\theta-2 \pi k),
$$

where $R_{m}, S_{m}$ and $T_{m}$ are geometric parameters and $\mathbb{1}_{[a, b]}$ denotes the indicator function of the interval $[a, b]$.

We now outline the application of Property 2 to these templates, using the fact that they are polar-separable.

1. $\boldsymbol{f}_{k}(r)$ is of the form $\mathbf{D}_{k} \tilde{\boldsymbol{f}}(r)$, where $\mathbf{D}_{k}$ is a diagonal matrix that can be computed using

$$
\frac{1}{2 \pi} \int_{-\pi}^{\pi} \mathrm{e}^{-\mathrm{i} k \theta} \mathbb{1}_{\left[-\frac{T}{2}, \frac{T}{2}\right]}(\theta) \mathrm{d} \theta=\frac{T}{2 \pi} \operatorname{sinc}\left(\frac{k T}{2 \pi}\right) .
$$

2. $\mathbf{H}_{k}=\mathbf{D}_{k}^{H} \widetilde{\mathbf{H}} \mathbf{D}_{k}$ where $\widetilde{\mathbf{H}}$ can be computed using

$$
\int_{0}^{+\infty} \mathbb{1}_{[R, S]}(r) \mathbb{1}_{\left[R^{\prime}, S^{\prime}\right]}(r) r \mathrm{~d} r=\frac{r_{\max }^{2}-r_{\min }^{2}}{2},
$$

with $r_{\min }=\max \left(R, R^{\prime}\right)$ and $r_{\max }=\min \left(S, S^{\prime}\right)$.

3. The eigenvectors and eigenvalues of the $4 \times 4$-matrices $\mathbf{H}_{k}$ can be computed numerically.

4. The resulting functions $\varphi_{n}=u_{n}$ are represented graphically in Fig. 2 for $N=24$.

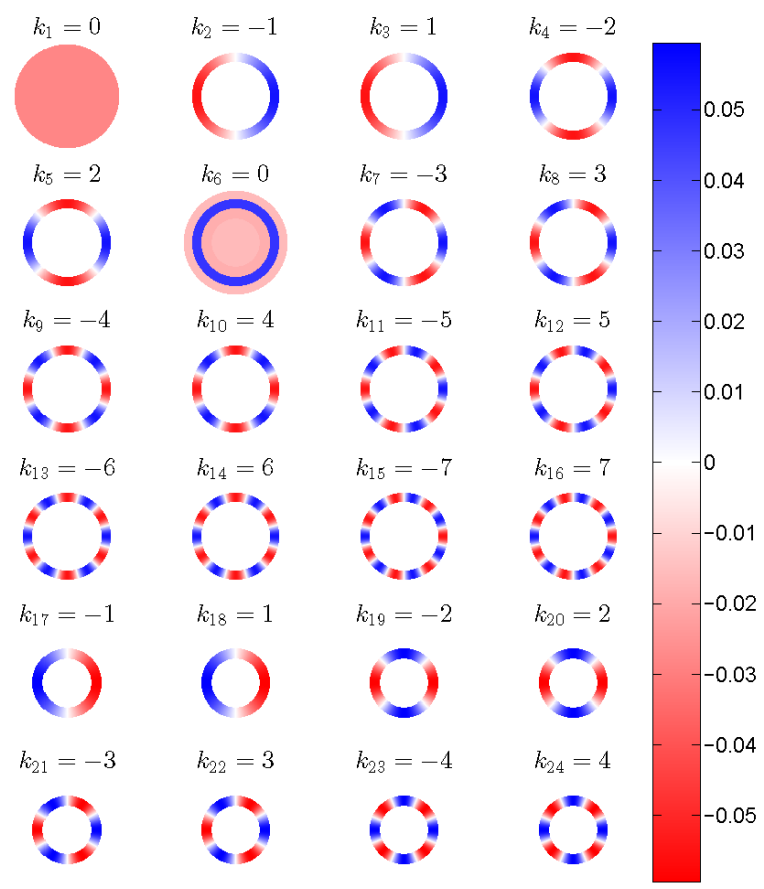

Fig. 2. The real part of the first 24 principal components, with the corresponding Fourier-series harmonics $k_{n}$.

\subsection{Detection algorithm}

The particle-recognition algorithm comprises three main steps. We will need the following result.

Property 3. The best approximation of $R_{\theta}\{\boldsymbol{f}\}$ in the principalcomponent subspace is given by $\sum_{n=1}^{N}\left\langle\varphi_{n}, R_{\theta}\{\boldsymbol{f}\}\right\rangle \varphi_{n}$, where $\left\langle\varphi_{n}, R_{\theta}\{\boldsymbol{f}\}\right\rangle=\sqrt{\lambda_{n} / 2 \pi} e^{-\mathrm{i} k_{n} \theta} \mathbf{v}_{n}$

First the particles are detected and localized. This is done by finding the local extrema of the correlation between the input image and a template of the form

$$
w_{1} \mathbb{1}_{\left[0, R_{3}\right]}(r)+w_{2} \mathbb{1}_{\left[R_{3}, R_{2}\right]}(r)+w_{3} \mathbb{1}_{\left[S_{2}, S_{1}\right]}(r),
$$

where $w_{1}, w_{2}$ and $w_{3}$ denote weighting coefficients.

The next step is to determine the orientation $\theta_{0}$ of each particle. To this end we need the best approximation of the orientation mark in the steerable basis, hereafter denoted by $q=\sum_{n=1}^{N} q_{n} \varphi_{n}$. We steer $q$ so as to maximize the correlation with the particle. Owing to (1), $\left\langle R_{\theta_{0}}\{p\}, R_{\theta}\{q\}\right\rangle=\sum_{n=1}^{N} q_{n}\left\langle R_{\theta_{0}}\{p\}, \varphi_{n}\right\rangle \mathrm{e}^{-\mathrm{i} k_{n} \theta}$ is a trigonometric polynomial with respect to $\theta$, which can be maximized numerically. We emphasize that this step only involves inner products at the locations of the detected particles-there is no convolution with the entire image.

Finally the code must be extracted. At this stage we can subtract the contributions of the disk and of the orientation mark, leaving us with the final sum in (6). Based on Property 3 and Fig. 2, we can then observe that the inner products with the first 14 principal 

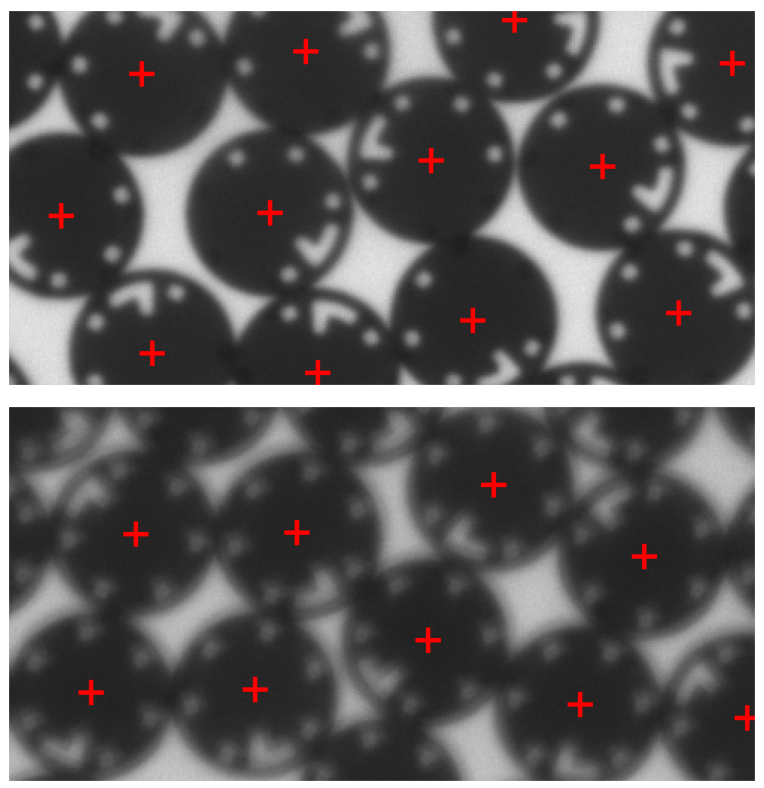

Fig. 3. Images of real micro-particles. The red crosses indicate the detected locations of the particles.

components give us access to the DFT of the code:

$$
\left\langle\varphi_{n}, \sum_{\ell=-5}^{5} c[\ell] R_{\frac{2 \pi \ell}{13}}\left\{f_{4}\right\}\right\rangle=\sqrt{\frac{\lambda_{n}}{2 \pi}} \mathbf{v}^{T} \mathbf{v}_{n} \sum_{\ell=-5}^{5} \mathrm{e}^{-\mathrm{i} 2 \pi k_{n} \ell / 13} c[\ell],
$$

where $\mathbf{v}^{T}=\left[\begin{array}{llll}0 & 0 & 0 & 1\end{array}\right]$ and $k_{n} \in \llbracket-6,6 \rrbracket$. Thus the code can be recovered using an FFT-based algorithm, after a deconvolution step to compensate for the multiplicative constants.

\subsection{Numerical results}

We first tested the algorithm on a real image containing 229 particles. The original size of the image was $2592 \times 1944$; a small region of interest is shown in Fig. 3 (top). All particles were correctly detected (no false positives or negatives) and more than $99 \%$ of the codes were correctly recognized. The computation time on a laptop computer with a $2.6 \mathrm{GHz}$ CPU was approximately 7 seconds, without parallelizing the code. Similar detection rates were achieved with another class of particles under less favorable focusing conditions (Fig. 3, bottom).

Since in the real images we had at our disposal all micro-carriers had the same perforations (up to reflection), we also tested our algorithm on a set of 50 synthetic images. Each image contained a particle whose orientation and code were drawn randomly from a uniform distribution. In addition, to simulate optical defocus, the images were convolved with a round uniform-blur kernel whose diameter was 6 pixels. The results are reported in Table 1. It is seen that the position and orientation estimates are accurate enough to allow for zero decoding errors.

\section{CONCLUSION}

Motivated by an application in molecular diagnostics, we have presented a PCA-based procedure for designing steerable filters so as

\begin{tabular}{|r||c|c||c|}
\hline error & position (pixels) & orientation (radians) & code $(\%)$ \\
\hline \hline mean & 1.2251 & 0.0118 & 0 \\
std dev & 0.6349 & 0.0097 & 0 \\
\hline
\end{tabular}

Table 1. Position, orientation and code error for a set of 50 synthetic images.

to best approximate a given set of templates. The specificity of our framework is that it is formulated in the continuous domain and allows for multiple templates.

Furthermore we have developed a method that harnesses the adaptivity and the computational efficiency of our steerable filters for detecting and classifying micro-particles used in binding experiments. Our method can deliver feedback on the reaction within a few seconds, which is important if the lab operator needs to terminate the data-acquisition process before completion.

Engineers at Biocartis have praised the detection algorithm for its elegance and computational efficiency; it is currently being further developed by the company.

\section{ACKNOWLEDGMENTS}

The authors would like to thank Mathieu Gaillard, Nicolas Demierre and José Gil from Biocartis for the fruitful collaboration on this project. This work was funded in part by ERC Grant ERC-2010AdG 267439-FUN-SP.

\section{REFERENCES}

[1] P. Perona, "Steerable-scalable kernels for edge detection and junction analysis," Image and Vision Computing, vol. 10, no. 10, pp. 663-672, 1992.

[2] P. Perona, "Deformable kernels for early vision," IEEE Transactions on Pattern Analysis and Machine Intelligence, vol. 17, no. 5, pp. 488-499, May 1995.

[3] G. Sommer, M. Michaelis, and R. Herpers, "The SVD approach for steerable filter design," in Proceedings of the 1998 IEEE International Symposium on Circuits and Systems, May 31-June 3, 1998, vol. 5, pp. 349-353.

[4] M. Michaelis and G. Sommer, "A Lie group approach to steerable filters," Pattern Recognition Letters, vol. 16, no. 11, pp. 1165-1174, 1995.

[5] Y. Hel-Or and P. C. Teo, "Canonical decomposition of steerable functions," Journal of Mathematical Imaging and Vision, vol. 9, pp. 83-95, 1998.

[6] M. Unser and N. Chenouard, "A unifying parametric framework for 2D steerable wavelet transforms," SIAM Journal on Imaging Sciences, 2012, in press.

[7] Z. Püspöki, C. Vonesch, and M. Unser, "Detection of symmetric junctions in biological images using 2-D steerable wavelet transforms," Proceedings of the Tenth IEEE International Symposium on Biomedical Imaging: From Nano to Macro (ISBI'13), 2013.

[8] P. C. Teo and Y. Hel-Or, "Lie generators for computing steerable functions," Pattern Recognition Letters, vol. 19, no. 1, pp. 7-17, 1998.

[9] C. Ponce and A. Singer, "Computing steerable principal components of a large set of images and their rotations," IEEE Transactions on Image Processing, vol. 20, no. 11, pp. 3051-3062, November 2011.

[10] W. T. Freeman and E. H. Adelson, "The design and use of steerable filters," IEEE Transactions on Pattern Analysis and Machine Intelligence, vol. 13, no. 9, pp. 891-906, September 1991. 